\section{USO DE LOS POTENCIALES RELACIONADOS A EVENTOS COGNITIVOS (PRES) EN EL DIAGNÓSTICO DE TRASTORNO POR DÉFICIT DE LA ATENCIÓN E HIPERACTIVIDAD (TDAH)}

\section{USE OF EVENT-RELATED COGNITIVE POTENTIALS (ERP) IN THE DIAGNOSIS OF ATTENTION DEFICIT HYPERACTIVITY DISORDER (ADHD) ${ }^{1}$}

\begin{abstract}
Oscar Hernán Moscoso Ariza. BSc, MEd, PhD. Grupo de Neuroaprendizaje de la Universidad Autónoma de Manizales. ${ }^{2}$

Comentarios: oscarhma@autonoma.edu.co

Francia Restrepo de Mejía. MD, PhD. Grupo de Neuroaprendizaje de la Universidad Autónoma de Manizales. ${ }^{3}$

Mary Orrego Cardozo, PhD. Grupo BIMSA de la Universidad Autónoma de Manizales. ${ }^{4}$

José Armando Vidarte Claros, PhD. Grupo de Cuerpo Movimiento de la Universidad Autónoma de Manizales. ${ }^{5}$

Departamento de Ciencias Básicas Biológicas, Universidad Autónoma de Manizales. Grupo de Neuroaprendizaje, Línea Neurociencia Cognitiva.
\end{abstract}

Palabras clave: Atención, Hiperactividad, Trastorno de la Atención, Potenciales Cognitivos de Eventos Relacionados con.

Keywords: Attention, Hyperactivity, Disorder Attention, EventRelated Cognitive Potentials.

\section{RESUMEN}

Lo expuesto a continuación es una revisión sobre el trastorno por Déficit de la Atención e Hiperactividad el cual representa, entre otros, uno de los aspectos relevantes para la fundamentación teórica y el avance de la neurociencia cognitiva en este campo en particular, toda vez que los procesos de atención y memoria subyacen en la fisiopatología del sistema nervioso humano. El TDAH
1.Traducción: Blanca Stella Giraldo.

Revisión: Centro de Traducción del Instituto de Idiomas UAM

2.Licenciado en Biología de la UPN, especialista en Informática Educativa, Universidad de Manizales, magister en Educación, Universidad Pontificia J averiana. Ph D. en Neurociencia y Biología del Comportamiento, Pablo de Olaride, Sevilla España.

3.Médica fisiatra y doctora en Ciencias Sociales, Niñez y $\mathrm{J} \mathrm{u}$ ven t u d, C i n d e. Universidad de Manizales. Directora del laboratorio en Neuro Rehabilitación, UAM

4. Licenciada en Biología y Química por la Universidad de Caldas. Maestría y doctorado en Bioquímica y Biología Molecular por la Universidad Autónoma de B arcelona, España . Coordinadora del grupo de Investigación en Biología Molecular y Salud -BIMSAde la Universidad Autónoma de Manizales UAM. Profesora asociada de la UAM. Fue profesora asociada de la Universidad Autónoma de Barcelona 1999-2003. C atedrática de la Universidad Nacional de Colombia sede Manizales.

5. $\mathrm{PhD}$ en Ciencias de la Actividad Física y el Deporte. Profesor titular de la Universidad Autónoma de Manizales. Líder del Grupo de Investigación CuerpoMovimiento. UAM

Recibido: febrero 15 de 2010

Aprobado: abril 20 de 2010

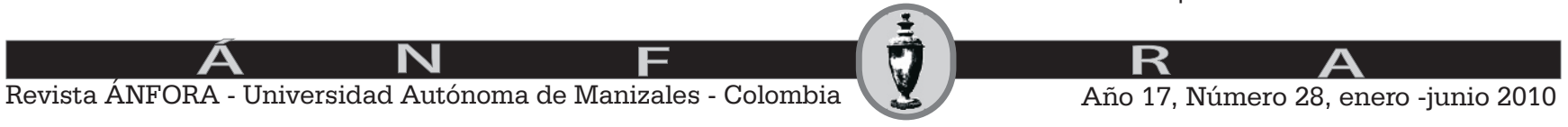


es una de las patologías crónicas mas frecuentes en la infancia, se presenta su definición, epidemiología, diagnóstico y tratamiento, y el uso de las técnicas neurofisiológicas denominadas Potenciales relacionados a eventos cognitivos para la detección temprana y para mejorar el diagnóstico diferencial y los procesos de intervención, reduciendo así el impacto negativo que causa en nuestra sociedad.

\section{ABSTRACT}

This paper presents a review of the Attention Deficit Hyperactivity Disorder. This represents one of the most relevant aspects for the theoretical basis and the advance of the cognitive neuroscience in this field. This is true because the attention and memory processes underlie the physiopathology of the central nervous system. The ADHD is one of the most frequent chronic diseases in childhood. We present here its definition, epidemiology, diagnosis and treatment. Moreover, we show the use of the neurophysiological techniques called event-related cognitive potentials for the early detection and the improvement of the differential diagnosis and the intervention processes, reducing thus the negative impact caused in our society.

\section{Introducción}

La Neurociencia está reconocida como pilar que fundamenta desde los aspectos teóricos, prácticos y experimentales el desarrollo y la comprensión de la Ciencia Cognitiva.

El objetivo es comprender la naturaleza y estructura de nuestras operaciones mentales. Las actividades mentales se describen en subsistemas de procesamiento requeridos para la realización de cada una de las etapas elementales necesarias, a fin de llevar a cabo una actividad mental particular, como leer una palabra o una oración, identificar un objeto percibido visualmente, resolver un problema, etc. La identificación de estos subsistemas descansa sobre hechos ligados a la anatomía y funcionamiento del cerebro.

En consecuencia, es preciso contar con datos sobre el cerebro para caracterizar las actividades mentales. No se trata de reemplazar una descripción de las actividades cognitivas por una descripción de las actividades del cerebro, pero

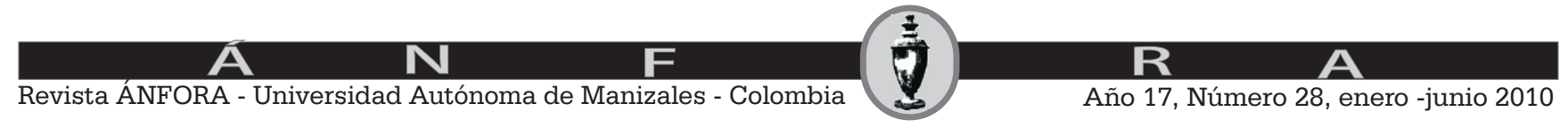


se postula que el pensamiento no brota de cualquier sustrato, y que su sustrato, el cerebro, condiciona las formas posibles que puede tomar el pensamiento. "Puede ser que la mente humana sea demasiado compleja para ser comprendida por la misma mente humana, pero el deseo de intentar lo posible parece ser una de sus características más persistentes" Slogan (1985)

El tema que se desarrolla a continuación es relevante en la ciencia cognitiva puesto que describe una alteración de una de las funciones cognitivas indispensables en el aprendizaje como es la atención, además que impacta todas las dimensiones del ser humano.

\section{DESARROLLO}

El Trastorno por Déficit de la Atención e Hiperactividad (TDAH) es una de las psicopatologías más frecuente en la infancia. Su prevalencia actual a nivel mundial se estima en un 5.29 \% (Polanczyk G., et al 2007); sin embargo, estudios realizados en Colombia basados en evaluación psiquiátrica estructurada, pruebas clínicas y test neuropsicológicos, estiman que alrededor del 17\% de la población entre los 4 y los 17 años de edad lo presentan (Pineda D. A., et al 1999, Pineda D. A., et al 2001 y Pineda D. A., et al 2003). Este trastorno está relacionado con problemas académicos, sociales, familiares y vocacionales tanto en los períodos de infancia y adolescencia, como en la adultez. Si no es diagnosticado correcta y precozmente, se asocia en la niñez con más altas tasas de deserción y fracaso escolar; y en la edad adulta con trastornos de conducta, alcoholismo y fármaco dependencia (International Consensus Statement on ADHD, 2002).

El TDAH se caracteriza por fallas en la atención, impulsividad e hiperactividad que afectan sustancialmente el funcionamiento cognitivo y comportamental de quien lo padece. Los recientes desarrollos en neuroimagenología, neurofisiología y genética han permitido un mejor conocimiento de la su etiología, entre las que se han identificado: alteraciones cromosómicas, lesiones cerebrales, disfunción de neurotransmisores y factores medioambientales (Castellanos F. X., Tannock R. 2002), aunque estas asociaciones no han sido confirmadas plenamente.

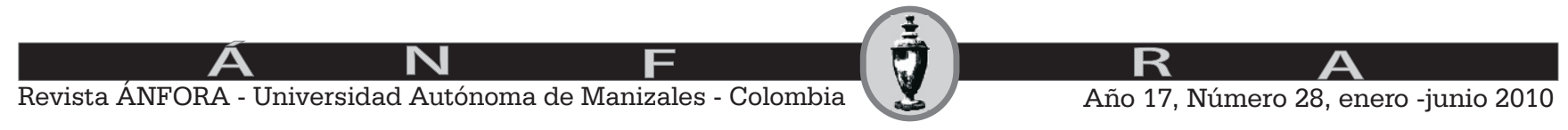


En su fisiopatología cobran relevancia los circuitos cerebrales relacionados con la atención y el procesamiento cerebral de la información (Malone M. A., et al 1994); señalando una lentificación en los tiempos de reacción cognitivos en los niños con TDAH.

El diagnóstico actual del TDAH se basa en una evaluación clínica especializada basada en los criterios del DSM-IV-TR y en los resultados de pruebas neuropsicológicas que evalúan la conducta de los individuos que puedan estar presentando esta alteración (American Psychiatric Association. DSM-IV-TR, 2000). Los resultados de dichos estudios conductuales indican que los niños con TDAH estadísticamente muestran menor cantidad de aciertos y mayor cantidad de errores de comisión o falsas alarmas que los controles en gran número de tareas cognitivas y atencionales (Idiazábal M. A., et al 2002); también demuestran que las diferencias de rendimiento entre ambos grupos se deben a un fallo en el funcionamiento ejecutivo del procesamiento (Jonkman L. M., et al. 1997). Las limitaciones de los estudios conductuales se deben a que en las medidas del rendimiento sólo se manifiesta el resultado final del procesamiento de la información (Idiazábal M. A., et al 2002). Las conclusiones basadas solamente en estos datos, pueden subestimar los déficits específicos del desorden, debido a que el procesamiento encubierto puede medirse indirectamente y con una resolución de tiempo limitada (Banaschewski T. et al 2003). Diferentes mecanismos encubiertos pueden llevar a un desempeño aparente indistinguible y así aparecer como tal, y un procesamiento central anómalo puede preceder a déficit de desempeño aparente o incluso estar asociado con desempeño normal (Brandeis et al., 1998; Taylor M. J., et al 1993; van Leeuwen et al., 1998).

\section{Definición}

El Trastorno por Déficit de la Atención e Hiperactividad (TDAH) es una entidad psicopatológica que se caracteriza por un patrón persistente de desatención y/o hiperactividad consideradas inapropiadas para la edad de desarrollo. Este patrón de comportamiento debe estar presente antes de los siete años de edad y manifestarse por un espacio de tiempo no inferior a 6 meses y presentarse por lo menos en dos ámbitos distintos (American Psychiatric Association, DSM-IVTR, 2000).

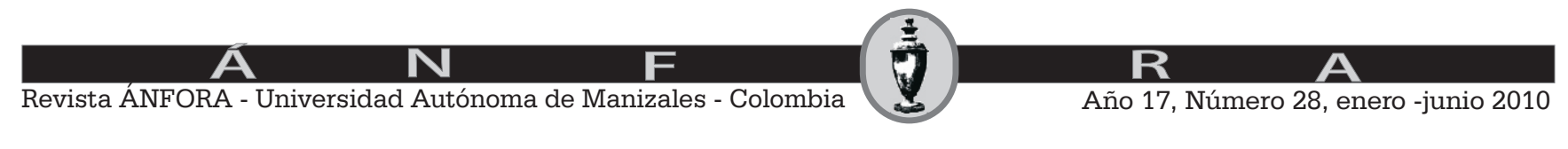




\section{Epidemiología}

La prevalencia en población manizalita se estima en 17\% en niños con edades comprendidas entre los 4 y los 17 años (Pineda D. A., et al 1999, Pineda D. A., et al 2001 y Pineda D. A., et al 2003). La razón de presentación por género masculino: femenino según estos estudios es de $3: 1$, sin diferencias de estrato. Según el subtipo, en la población colombiana la forma más frecuente es el combinado con $9.2 \%$, le sigue el predominantemente inatento con el $6.2 \%$ y el hiperactivo-impulsivo en último lugar con el 1\% (Pineda D. A., 2003).

\section{Diagnóstico}

Los primeros registros sobre niños hiperactivos aparecieron en la segunda mitad del siglo XIX, y la primera descripción del trastorno data de principios del siglo XX cuando un pediatra inglés presentó 20 casos de niños con hiperactividad, dificultad para sostener la atención, reacciones violentas, agresividad y poca respuesta al castigo, que interpretó como un defecto del control moral (Still G. F. 1902). En los siguientes cincuenta años, los mayores conocimientos en neurociencia y la experiencia adquirida en lesionados de guerra cambiaron la interpretación y se jerarquizó la hiperactividad como el síntoma dominante. En la década de los 60 Clements (Clements S. D. 1966) introduce el término de Disfunción Cerebral Mínima para referirse a los niños con manifestaciones de hiperactividad, deficiencia de la atención, e impulsividad, denominación que no gozó de apoyo empírico. En 1968 con el DSM-II se introdujo a la hiperactividad como un trastorno del comportamiento. En las décadas de los 70 y 80 los trabajos de Virginia Douglas (Douglas V. 1972) sobre los trastornos de hiperactividad en la infancia sirvieron para acuñar el término "trastorno por déficit atencional con y sin hiperactividad" utilizados en el DSM-III. Entre otros aspectos, la autora utilizó tareas de laboratorio para concluir que el principal problema de los niños con hiperactividad no era la distracción (y, por tanto, la selectividad atencional) sino el mantenimiento (es decir, la inatención sostenida). A principios de los años 90, Barkley desarrolló la idea de que entre los distintos subtipos de hiperactividad y en la diferenciación entre este y otros trastornos podía tener incidencia el tipo de disfunción atencional: los niños con TDA de predominio inatento podrían tener más problemas de focalización y velocidad de procesamiento, mientras los niños con TDA de predominio hiperactivo podrían tener más problemas de inatención

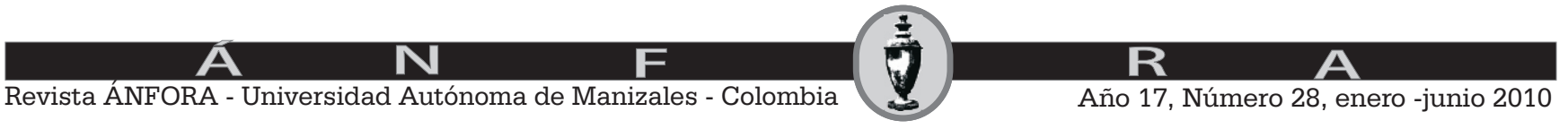


sostenida como reflejo de un problema de autocontrol (Barkley R. A. 1990 y Barkley R. A. 1997). El grupo de Van der Meere (Van der Meere. 1996) ha relacionado el rendimiento específico en tareas de atención sostenida con hipótesis explicativas del TDAH en el ámbito del sistema atencional de alertamiento (arousal) y la activación cortical.

Actualmente se considera como estándar de oro en el diagnóstico (Casado S. E. 2001) una entrevista psiquiátrica estructurada que está basado en 18 síntomas presentados como criterios por el DSM-IV-TR y el CIE-10 en forma similar (Miranda A., et al 1999). Aparte de los criterios clínicos y estadísticos, en el diagnóstico del TDAH se emplean varias escalas validadas Conners para profesores (Teachers Rate Scale) y para padres (Parents Rate Scale) (Farré-Riva A. y Narbona J. 1997).

\section{Comorbilidades}

El TDAH tiene una alta comorbilidad con patologías psiquiátricas como los trastornos conductuales disocial y negativista desafiante (40-50\%), trastornos de ansiedad (25\%), depresión (30-33\%), trastornos del aprendizaje (20-25\%) y síndrome de Gilles de la Tourette (2\%) (Calderón Ocampo J. H. y Amézquita Medina, M. E. 2001).

\section{Tratamiento}

Hacer un buen diagnóstico es el punto inicial del tratamiento. El manejo del TDAH es multidisciplinario y multicomponencial (Villar Alvarez L. F., 2001) involucrando a profesionales en psiquiatría, rehabilitación, psicología, fisioterapia, terapia ocupacional, fonoaudiología, educadores especiales, además de padres, maestros y familiares.

El tratamiento farmacológico favorece el mejoramiento de la atención selectiva permitiendo que las terapias y la nivelación pedagógica tengan su máximo rendimiento. Como fármacos de primera línea se encuentran los psicoestimulantes (metilfenidato principalmente) y los inhibidores selectivos de la recaptación de noradrenalina (Spencer T. J., et al 2002 y Pliszka S. R. 2003)

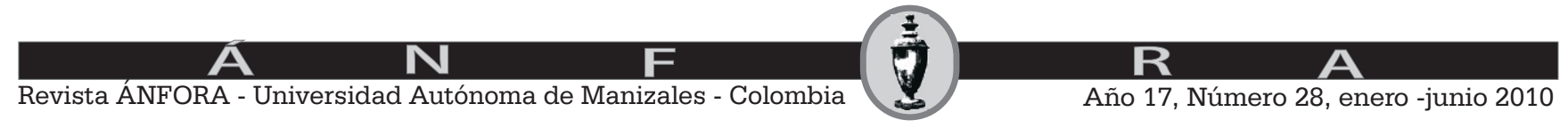




\section{Importancia del trastorno}

El TDAH se considera uno de los desórdenes del comportamiento más comunes de la infancia estimándose que más del $80 \%$ de los niños que lo presentan continuarán expresándolo en la adolescencia, etapa en la cual quienes lo padecen están mucho más propensos a retirarse de la escuela (32-40\%), a comprometerse en actividades antisociales (40-50\%), a hacer uso de drogas ilícitas más de lo normal, y a experimentar embarazo a una edad más temprana (40\%). Entre el 30\% y el 65\% de quienes fueron diagnosticados con esta enfermedad en la infancia lo presentarán también en la edad adulta, etapa en la cual se caracteriza por dificultades en las relaciones interpersonales, laborales y sociales y por su alta co-morbilidad con trastorno de personalidad antisocial farmacodependencia y alcoholismo (International Consensus Statement on ADHD, 2002).

\section{La atención}

La atención no puede reducirse a una única definición ni puede ligarse a una única estructura anatómica o evaluarse con un único test o prueba. Es útil considerar la atención como un proceso o capacidad multidimensional, cuyos diferentes componentes pueden ser sensibles a diferentes agentes. Con frecuencia, se habla de la atención selectiva, de la capacidad de dividir la atención y de aspectos intensivos del mantenimiento de la atención como la atención sostenida y la vigilancia. Recientemente se alude a otra acepción de la atención como mecanismo de control de la ejecución, y a la especificidad de mecanismos como los de enfoque, movimiento y desenganche de la atención en el espacio visual.

Los tipos tradicionalmente estudiados de la atención son:

- Atención selectiva. Es la capacidad para seleccionar, de entre varias posibles, la información relevante que se va a procesar o el esquema de acción apropiado.

- Atención dividida. Es la capacidad de realizar la selección de más de una información a la vez o de más de un proceso o esquema de acción simultáneamente. Estudia el proceso de compartir la capacidad entre tareas o fases de una tarea.

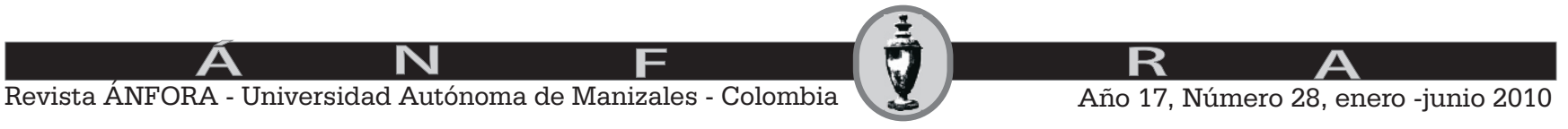


- Atención sostenida. Es la capacidad de mantener el estado de selectividad atencional durante un período prolongado en la realización de una tarea. Se habla de vigilancia cuando la tarea es de detección y de concentración cuando se refiere a otras tareas cognitivas.

Algunos autores (Van der Heijden A. H. C., 1992) consideran la atención como el estado cognitivo dinámico que favorece el comportamiento selectivo en una situación específica de tarea. Es la selección de la información (externa o interna) relevante a la situación o la selección del proceso cognitivo o respuesta motriz adecuada para la acción. La atención consiste en la distribución de valores de activación sobre esquemas y acciones; asimismo, posibilita la adecuada orientación del comportamiento a los requisitos de tarea. Tales requisitos pueden hacer que en una situación concreta se necesite enfatizar la selectividad atencional, en otra la capacidad de compartir la atención entre varias tareas o fases de tarea, en otra el mantenimiento de la atención y la concentración y, en último extremo, encontramos la atención como mecanismo supervisor de la ejecución llevada a cabo. Esto es, se trataría del mecanismo cognitivo mediante el cual se ejercería un control voluntario sobre la actividad perceptiva, cognitiva y comportamental, cuando ésta no pudiera llevarse a cabo de manera automática (García-Ogueta M. I. 2001).

\section{Bases bioeléctricas de los potenciales relacionados a eventos cognitivos (PREs)}

\section{Potencial de acción neuronal}

El potencial de acción neuronal o impulso nervioso puede considerarse como una onda eléctrica negativa de autopropagación que avanza sobre la superficie de la membrana de la neurona y de sus prolongaciones. Esta propiedad de las neuronas se debe a su capacidad de generar gradientes iónicos tanto en el interior como en el exterior de la membrana celular. Al producirse un estímulo, la neurona se hace más permeable a los iones de sodio, los cuales entran a la célula, saliendo al exterior iones de potasio. Este movimiento iónico hace que la superficie interna de la membrana celular se vuelva positiva en relación con la superficie externa. Éste cambio bioeléctrico se conoce como despolarización, la cual se propaga a todo lo largo de la neurona y sus prolongaciones (Kandel E., et al 1997).

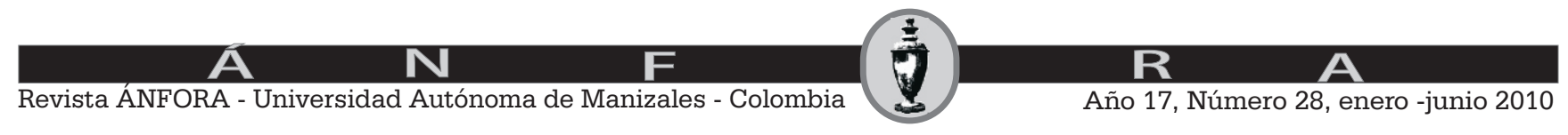




\section{Registro de los potenciales de acción}

Estos acontecimientos bioeléctricos pueden registrarse por medio de electrodos no invasivos conectados a un amplificador de voltaje (Smith J. L., et al 2003), que se colocan sobre el cuero cabelludo de acuerdo al sistema internacional 10-20 (Jasper H. H., 1958). Los cambios en la actividad bioeléctrica cerebral durante la presentación de estímulos de una región particular pueden utilizarse para determinar la integridad de una vía nerviosa, o si aquella interviene en funciones cognitivas superiores.

Los PREs son fluctuaciones en el voltaje cerebral que se miden sobre el cuero cabelludo y que se obtienen promediando la respuesta cerebral a un estímulo determinado (visual, auditivo, somatosensorial o cognitivo) sobre un número determinado de pruebas (Chiapa K. H. 1989, Banaschewski T., et al 2003). Sus componentes se caracterizan por poseer una polaridad, una topografía, una amplitud, una latencia y una relación específica con las variables de la tarea que los genera, los cuales se denominan ondas N100, P200, N200 y P300. Las variaciones en la amplitud reflejan de manera primaria una variación en la intensidad de la activación de las estructuras corticales, representando los aspectos energéticos del procesamiento, mientras que la latencia de un componente se relaciona con la velocidad de procesamiento de la información (Mulder G. 1986).

\section{Uso de los potenciales evocados en el TDAH}

Los PREs se han empleado como marcadores biológicos del TDAH desde la década de 1970 (Satterfield J. H. et al., 1972). Los objetivos de muchos de estos estudios han sido la medición de la amplitud y la latencia de la onda P300, examinar los cambios electroencefalográficos en las ondas N100, N200, y P200 y comprobar si la ejecución en las tareas atencionales de los niños con TDAH es significativamente menor que la de los niños control. La mayoría de los estudios han puesto de manifiesto la existencia de alteraciones significativas en los PREs en niños con TDAH al compararlos con niños normales, manifestadas como una disminución de la amplitud y un incremento de la latencia de los componentes estudiados (Barry R. J. et al. 2003b).

Un tipo de estudio hacia el cual se orienta gran parte de los esfuerzos para caracterizar el TDAH son los electrofisiológicos con el uso los potenciales 
relacionados a eventos cognitivos (PREs). Los PREs representan el correlato eléctrico sincrónico de los procesos cerebrales subyacentes a un estímulo presentado al sujeto (Chiapa K. H. 1989), y sus componentes se han asociado con el nivel de procesamiento de dicho estímulo. Los PREs pueden revelar los precursores psicofisiológicos y los correlatos de los desempeños en diversas pruebas, y de este modo revelar diferencias específicas en el procesamiento encubierto de la información en ausencia de diferencias en el desempeño (Brandeis D. et al., 1998; van Leeuwen T. H. et al., 1998).

Uno de los primeros componentes endógenos descritos y relacionado con tareas atencionales es una negatividad temprana nombrada N100, éste representa la extracción inicial de información sensorial del estímulo (Näätänen R. y Picton T. W. 1987) o la excitación eléctrica asociada con el empleo de un canal para el procesamiento de la información por fuera de la corteza de asociación primaria (Hansen J. C. y Hillyard S. A. 1988 y Oades R. D. 1998). Otro de los componentes relacionado con la atención sensorial selectiva a estímulos visuales es la onda P200 (Hegerl and Juckel, 1993), la cual representa la inhibición del input sensorial mediante la identificación automática del estímulo y la posterior discriminación/clasificación del mismo (Lindholm y Koriath, 1985), o inhibición de otros canales de información que compiten por atención y mas procesamiento (Hansen J. C. y Hillyard S. A., 1988; Oades R. D., 1998); por su parte la onda N200, es un componente que se relaciona con la discriminación de estímulos, el reconocimiento de señales de parada en tareas de tipo go no-go, además con el procesamiento discriminante del estímulo, y con el proceso de detección de incompatibilidades en el mismo (Näätänen R. y Picton T. W. 1986); por ultimo, la onda P300, se relaciona con la actualización contextual y los procesos de categorización/discriminación del estímulo, específicamente con el proceso cognitivo necesario para actualizar el modelo mental del contexto al interior de la memoria de trabajo (Donchin E. y Coles M. G. 1988); se ha propuesto además que este componente es un marcador del almacenamiento de memoria así como un enlace entre las características del estímulo y la atención prestada al estímulo novedoso (Näätänen R., 1990).

Existe evidencia de que los componentes de los PREs tanto en tareas visuales como auditivas difieren entre niños con TDAH y niños control en los rangos de latencia entre los 100 y los 250 milisegundos (Jonkman et al. 1997; Robaey et al. 1992; Yordanova et al. 2000). Se ha demostrado, tanto en adultos como en niños,

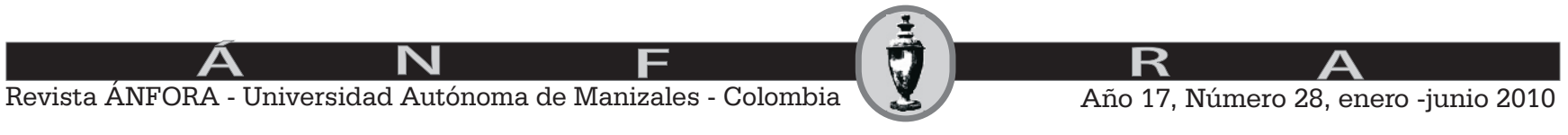


que las funciones atencionales pueden modular estas etapas tempranas del procesamiento perceptual/cognitivo. Las latencias de los componentes tempranos son típicamente más cortas en niños con TDAH que en los controles (Robaey et al., 1992; Satterfield et al., 1994), sugiriendo que los pacientes con TDAH procesan la información perceptual más rápido durante las primeras fases. Mientras que durante las fases más tardías de procesamiento tendrían un fallo para la localización de los recursos atencionales suficientes para llevar a cabo la tarea como se demuestra por la prolongación y menor amplitud de los componentes N200 y P300 (Banaschewski T., et al 2003, Vera et al 2007).

Lo anteriormente expuesto demuestra que el desarrollo de investigaciones y construcción de conocimiento en el campo de la atención, la memoria y sus alteraciones, es uno de los problemas prioritarios en la línea de neurociencia cognitiva.

\section{Conclusion}

El carácter multifactorial del trastorno, y su alto costo socioeconómico, exigen la búsqueda de indicadores neurofisiológicos que permitan una mejor identificación de los niños que requieren tratamiento y apoyo especial de acuerdo con su problema específico.

\section{Referencias}

American Academy of Child and Adolescent Psychiatry (2007). Practice Parameter for the Assessment and Treatment of Children and Adolescents With Attention-Deficit/Hyperactivity Disorder. J Am Acad Child Adolesc Psychiatry, 46:7, 894-921.

American Academy of Pediatrics (2001). Clinical practice guideline: treatment of the school-aged child with attention-deficit/hyperactivity disorder. Pediatrics 108:1033-1044.

American Psychiatric Association (2000), Diagnostic and Statistical Manual of Mental Disorders, 4th edition, Text Revision (DSM-IV-TR). Washington, DC:

American Psychiatric Association.

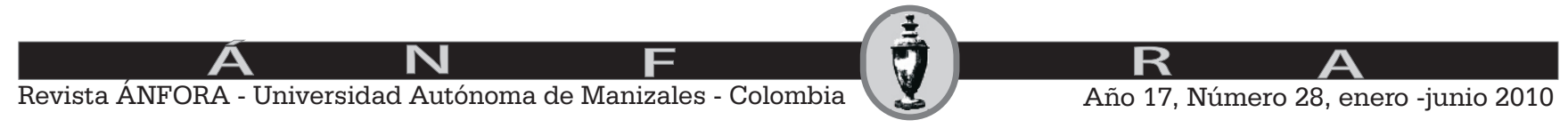


BANASCHEWSKI T., BRANDEIS D., HEINRICH H., ALBRECHT B., BRUNNER E., y ROTHENBERGER A. (2003). Association of ADHD and conduct disorder - brain electrical evidence for the existence of a distinct subtype. Journal of Child Psychology and Psychiatry 44 (3),356-376.

BARKLEY R. A. (1990) Attention deficit hyperactivity disorder: a handbook of diagnosis and treatment. New York: Guilford.

BARKLEY R. A. (1997) ADHD and the nature of selfcontrol. New York: Guilford.

BARRY R. J., Clarke A. R., Johnstone S. J. (2003a). A review of electrophysiology in attention-deficit/hyperactivity disorder: I. Qualitative and quantitative electroencephalography. Clin Neurophysiol;114:171-183.

BARRY R. J., JOHNSTONE S. J., CLARKE A. R. (2003b). A review of electrophysiology in attention-deficit/hyperactivity disorder: II. Event-related potentials. Clinical Neurophysiology 114 184-198.

BRANDEIS D., VAN LEEUWEN T.H., RUBIA K., VITACCO D., STEGER J., Pascual-Marqui R.D., y Steinhausen H.C. (1998). Neuroelectric mapping reveals precursor of stop failures in children with attention deficits. Behavioral Brain Research, 94, 111-125.

CALDERÓN OCAMPO J. H. y AMÉZOUITA MEDINA M. E. (2001). Elementos básicos para el abordaje de los trastornos mentales. Manizales, Colombia: Centro Editorial Universidad de Caldas; 541-563.

CASADO S. E. (2001). Déficit de atención con hiperactividad. aspectos generales. Rev Psiquiatr Psicol Niño y Adolesc, 4(1): 61-4.

CASTELLANOS F. X. y TANNOCK R. (2002). Neuroscience of attentiondeficit/hyperactivity disorder: The search for endophenotypes. Nature reviews neuroscience; (3): 617-622.

CHIAPA K. H., ed. (1989). Evoked potentials in clinical medicine. New York: Raven Press.

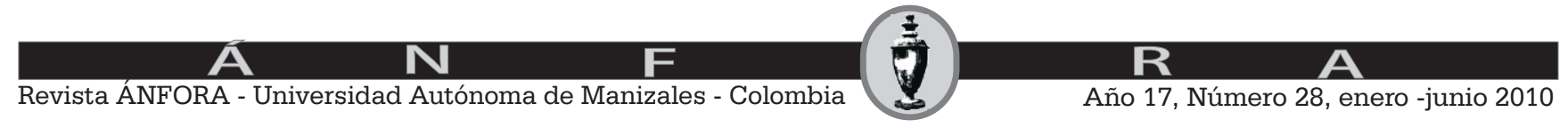


CLEMENTS S. D. (1966) Minimal brain dysfunction in children. Terminology and Identification (USPH Publication No. 1415). Washington: Government Printing Office.

CONNERS C. K. (1979). Conners teacher reading scale (hyperkinesis index). Chicago: Abbott Laboratories.

CORDES M. y MCLAUGHLIN T. F. (1998). Attention deficit hyperactivity disorder and rating scales with a brief review of the Connors teacher rating scale. International Journal of Special Education 2004; 19 (2): 23 - 34.

DONCHIN E., y COLES M. G. (1988). Is the P300 component a manifestation of context updating?. Behav Brain Sci;11:357-427.

DOUGLAS V. (1972). Stop, look and listen: the problem of sustained attention impulse control in hyperactive and normal childre. Can J Behav Sci; 4: 159 - 82.

DUPAUL G., POWER T. J., ANASTOPOULOS A. D. y REID R. (1998). ADHDRating Scales DSM-IV for parents and teachers. New York: Guilford.

FARRÉ-RIVA A. y NARBONA J. (1997) Escala de Conners en la evaluación del Trastorno por Déficit de Atención con Hiperactividad. Nuevo estudio de validación y análisis factorial en niños españoles. Rev Neurol; 25: 200-4.

García-Ogueta M. I. (2001). Mecanismos atencionales y síndromes neuropsicológicos. Rev Neurol; 32: 463-7.

HANSEN J. C. y HILLYARD S. A. (1988). Temporal dynamics of human auditory selective attention. Psychophysiology; 25:316-329.

HEGERL U. y JUCKEL G. (1993). Intensity dependence of auditory evoked potentials as an indicator of central serotonergic neurotransmission: a new hypothesis. Biol Psychiatry; 33:173-187.

IDIAZÁBAL M. A., PALENCIA-TABOADA A. B., SANGORRÍN J., ESPADALERGAMISSANS J. M. (2002). Potenciales evocados cognitivos en el trastorno por déficit de atención con hiperactividad. Rev Neurol; 34: 301-5.

International Consensus Statement on ADHD (2002). Clinical Child and Family Psychology Review; 5 (2): 89-111.

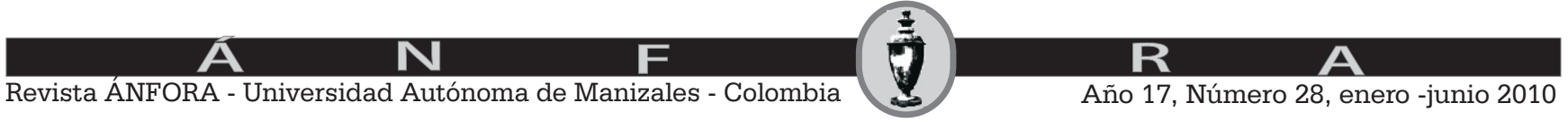


JASPER H. H. (1958). The ten-twenty electrode system of the International Federation. Electroencephalogr Clin Neurophysiol; 22: 497-507.

JONKMAN L. M., KEMNER C., VERBATEN M. N., KOELEGA H. S., CAMFFERMAN G., VD GAAG R. J., et al. (1997). Event related potentials and performance of attention deficit hyperactivity disorder: children and normals controls in auditory and visual selective attention tasks. Biol Psychiatry; 41: 595611

KANDEL R., ERIC, SCHWARTZ H., JAMES y JESSELL M., Thomas (1997). Neurociencia y Conducta. Prentice Hall. Madrid, págs. 175-193.

LINDEN D. (2005). The P300: Where in the Brain Is It Produced and What Does It Tell Us?. Neuroscientist 11(6):563-576.

LINDHOLM E. y KORIATH J. J. (1985). Analysis of multiple event related potential components in a tone discrimination task. Int $J$ Psychophysiol;3:121-129.

MALONE M. A., KERSHNER J. R., SWANSON J. M. (1994). Hemispheric processing and methylphenidate effects in attention-deficit hyperactivity disorder. Journal of Child Neurology, 9 (2): 181-9.

MIRANDA A., JAROUE S., SORIANO M. (1999). Trastorno de hiperactividad con déficit de atención: polémicas actuales acerca de su definición, epidemiología, bases etiológicas y aproximaciones a la intervención. Rev Neurol; 28 (Supl 2): S 182-8.

MULDER G. (1986). The concept and measurement of mental effort. In G.R.J. Hockey, A.W.K. Gaillard, \& M.G.H. Coles (Eds.), Energetics and human information processing (pp. 175-198)

NÄÄTÄNEN R. y PICTON T. W. (1986). N2 and automatic versus controlled processes. In: McCallum WC, Zappoli R, Denoth F, editors. Cerebral psychophysiology: studies in event-related potentials (EEG Suppl 38), Amsterdam: Elsevier, 169-186.

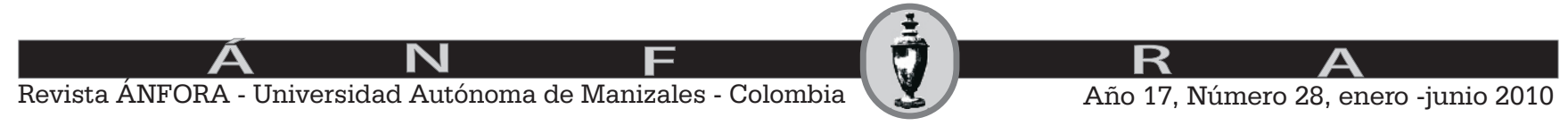


NÄÄTÄNEN R. y PICTON T. W. (1987). The N1 wave of the human electric and magnetic response to sound: a review and an analysis of the component structure. Psychophysiology;24:375-425.

NÄÄTÄNEN R. (1990). The role of attention in auditory information processing as revealed by event-related potentials and other brain measures of cognitive function. Behavioral and Brain Sciences; 13:201-288.

OADES R. D. (1998). Frontal, temporal and lateralized brain function in children with attention-deficit hyperactivity disorder: a psychophysiological and neuropsychological viewpoint on development. Behav Brain Res;94:83-95

OFFORD D. R. et al. (1996) Integrating assessment data from multiple informants. J. Am. Acad. Child Adolesc. Psychiatry 35, 1078-1085.

OZDAG M.F., YORBIK O., ULAS U.H., HAMAMCIOGLU K., y VURAL O (2004). Effect of methylphenidate on auditory event related potential in boys with attention deficit hyperactivity disorder. International Journal of Pediatric Otorhinolaryngology 68, 1267-1272.

PINEDA D., ARDILA A., ROSSELLI M., ARIAS B.E., HENAO G.C., GÓMEZ L.F., MEJÍA S.E. \& MIRANDA, M.L. (1999). Prevalence of attentiondeficit/hyperactivity disorder symptoms in 4 to 17-years-old children in the general population. Journal of Abnormal Child Psychology, 27, 455-462.

PINEDA D. A., LOPERA F., HENAO G. C., PALACIO J. D., CASTELLANOS F. X. (2001). Confirmación de la alta prevalencia del trastorno por déficit de la atención en una comunidad colombiana. Rev Neurol; 32(3):217-22.

PINEDA D. A., LOPERA F., PALACIO J. D., RAMIREZ D., HENAO G. C (2003). Estimaciones de la prevalencia del trastorno por déficit de la atención/hiperactividad: diagnósticos diferenciales y comorbilidades en una muestra colombiana. Int J Neurosci; 113 (1):49-71

PINEDA, David. Avances en la investigación del trastorno de atención con y sin hiperactividad. III Congreso Internacional de Cerebro y Mente; 2003 abril 22-25, Medellín, Colombia. Rev Neuropsicología, Neuropsiquiatría y Neurociencias 2003; 5 (1): 20-21

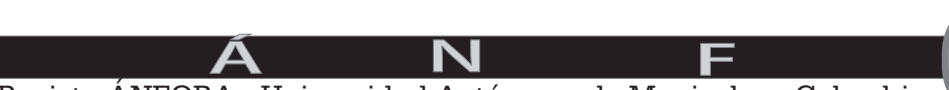


PLISZKA S. R. (2003). Non-stimulant treatment of attentiondeficit/hyperactivity disorder. CNS Spectr; 8(4):253-8.

POLANCZYK G., SILVA DE LIMA M., LESSA HORTA B., BIEDERMAN J., ROHDE L. A. (2007). The Worldwide Prevalence of ADHD: A Systematic Review and Metaregression Análisis. Am J Psychiatry; 164:942-948.

ROBAEY, P., BRETON, F., DUGAS, M., \& RENAULT, B. (1992). An event-related potential study of controlled and automatic processes in 6-8-year-old boys with attention deficit hyperactivity disorder. Electroencephalography and Clinical Neurophysiology, 82, 330-340.

SATTERFIELD J. H., CANTWELL D. P., LESSER L. I., PODOSIN R. L. (1972). Physiological studies of the hyperkinetic child. Am J Psychiatry;128:102-108.

SATTERFIELD, J.H., SCHELL, A.M., y NICHOLAS, T. (1994). Preferential neural processing of attended stimuli in attention-deficit hyperactivity disorder and normal boys. Psychophysiology, 31, 1-10.

SMITH J. L., JOHNSTONE S. J., y BARRY R. J. (2003). Aiding diagnosis of attention-deficit/hyperactivity disorder and its subtypes: discrimination function analysis of event-related potetnial data. Journal of Child Psychology and Psychiatry 44:7, 1067-1075.

SPENCER T. J., BIEDERMAN J., WILENS T. E., FARAONE S. V. (2002). Overview and neurobiology of attention deficit/hyperactivity disorder. J Clin Psychiatry; 63 (Suppl 12): 3-9.

SPITZER J.B. WILLIAMS M. y GIBBON M.B. (1990). First, Structured Clinical Interview for DSM-III-R: Non-Patient Edition (SCID-NP, Version 1.0), American Psychiatric Press, Washington, DC.

STILL G. F. (1902) Ths Coulstonian lectures: some abnormal physical conditions in children; Lecture II. Delivered before the Royal College of Physicians. London.

TAYLOR M. J., VOROS J. G., LOGAN W. J., y MALONE M.A. (1993). Changes in event-related potentials with stimulant medication in children with attention deficit hyperactivity disorder. Biological Psychology, 36, 139-156.

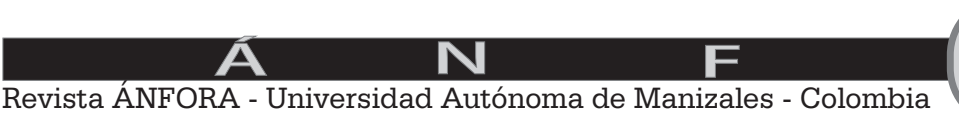


TODD R. D. y BOTTERON K. N. (2001) Is attention-deficit/hyperactivity disorder an energy deficiency syndrome? Biol. Psychiatry; 50: 151-158.

VAN DER HEIJDEN A. H. C. (1992). Selective attention in vision. London: Routledge.

VAN DER MEERE, J.J. (1996). The role of attention. En S. Sandberg (Ed.), Hyperactivity disorders of childhood (pp. 111-148). Cambridge: Cambridge University Press.

VAN LEEUWEN T. H., STEINHAUSEN H. C., OVERTOOM C. C., PASCUALMAROUI R. D., VAN'T KLOOSTER B., ROTHENBERGER A., SERGEANT J. A., y BRANDEIS D. (1998). The continuous performance test revisited with neuroelectric mapping: Impaired orienting in children with attention deficits. Behavioral Brain Research, 94, 97-110.

VERA A., RESTREPO F. y MOSCOSO O. H. (2007) Potenciales evocados cognitivos en trastorno por déficit atención/hiperactividad. Revista Neuropsicología, Neuropsiquiatría y Neurociencias; 7: 9-36.

VILLAR ALVAREZ L. F. (2001). Déficit De Atención Con/Sin Hiperactividad (ADHD): Tratamiento Farmacológico. Rev Psiquiatr Psicol Niño y Adolesc, 4(1): 103-110.

YORDANOVA J., KOLEV V., HEINRICH H., BANASCHEWSKI T., WOERNER W.,y ROTHENBERGER, A. (2000). Gamma band response in children is related to task-stimulus processing. Neuroreport, 11, 2325-2330.

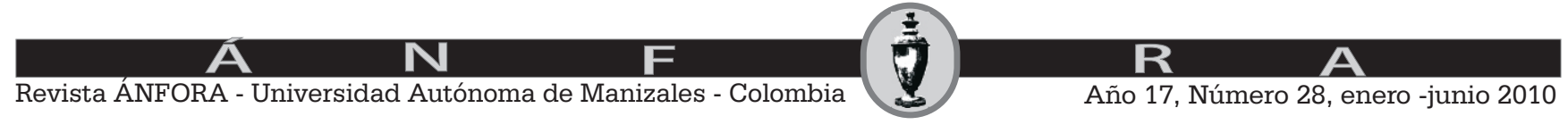

